\title{
A little bit of sex matters for genome evolution in asexual plants
}

\section{Diego Hojsgaard* and Elvira Hörandl*}

Department of Systematics, Biodiversity and Evolution of Plants, Albrecht-von-Haller Institute for Plant Sciences, Georg-August University of Göttingen, Göttingen, Germany

\section{Edited by:}

Ravishankar Palanivelu, University

of Arizona, USA

Reviewed by:

John E. Fowler, Oregon State

University, USA

Richard D. Noyes, University of

Central Arkansas, USA

*Correspondence:

Diego Hojsgaard and Elvira Hörandl, Department of Systematics,

Biodiversity and Evolution of Plants,

Albrecht-von-Haller Institute for

Plant Sciences, Georg-August

University of Göttingen, Untere

Karspüle 2, D-37073 Göttingen,

Germany

e-mail:diego.hojsgaard@

biologie.uni-goettingen.de;

elvira.hoerandl@

biologie.uni-goettingen.de
Genome evolution in asexual organisms is theoretically expected to be shaped by various factors: first, hybrid origin, and polyploidy confer a genomic constitution of highly heterozygous genotypes with multiple copies of genes; second, asexuality confers a lack of recombination and variation in populations, which reduces the efficiency of selection against deleterious mutations; hence, the accumulation of mutations and a gradual increase in mutational load (Muller's ratchet) would lead to rapid extinction of asexual lineages; third, allelic sequence divergence is expected to result in rapid divergence of lineages (Meselson effect). Recent transcriptome studies on the asexual polyploid complex Ranunculus auricomus using single-nucleotide polymorphisms confirmed neutral allelic sequence divergence within a short time frame, but rejected a hypothesis of a genomewide accumulation of mutations in asexuals compared to sexuals, except for a few genes related to reproductive development. We discuss a general model that the observed incidence of facultative sexuality in plants may unmask deleterious mutations with partial dominance and expose them efficiently to purging selection. A little bit of sex may help to avoid genomic decay and extinction.

Keywords: apomixis, Muller's ratchet, Meselson effect, polyploidy, heterozygosity

\section{INTRODUCTION}

Currently, the understanding of evolution patterns of genomes on different phylogenetic groups is a hot topic in evolutionary biology. The arrival of next generation sequencing (NGS) technologies and the generation of huge amounts of genomic data is allowing researchers to dig in the past and better resolve organisms' natural history as well as evolutionary enigmas. One of such enigmas is the predominance of sex in nature (Otto, 2009). One of the most prominent theories explaining the benefits of sex (for broad analyses see, e.g., Bell, 1982; Birdsell and Wills, 2003) proposes that sexuality protects the genome from the accumulation of deleterious mutations (Muller, 1964; Kondrashov, 1988; Hörandl, 2009; Figure 1). Here we will discuss theoretical assumptions and empirical possibilities of presence/absence of meiosis for asexual plant genome evolution in the light of unexpected recent findings on sexual/asexual taxa of Ranunculus.

Sexuality is a crucial factor molding the genomic features of eukaryotes. In plants, the formation of a new individual through sexuality involves an alternation between the sporophytic $(2 \mathrm{n})$ and the gametophytic (n) generations via meiosis and gamete fusion, the two mechanisms that create new genetic combinations. Additionally, outcrossing further potentiates genetic variation in populations. Thus, with few exceptions, every single sexual organism has a distinctive genotype that differentiates it from parents and siblings. Therefore, meiosis is the main source of genetic recombination and mixis, and segregates genetic factors in the offspring creating genetic variation. By doing so, meiosis and sexuality allows natural selection purging a lineage from harmful mutations. Because plant meiosis produces spores (mega- and microspores) and these spores develop into female and male gametophytes, in which considerable percentages of genes are being expressed (Joseph and Kirkpatrick, 2004), selection in a sexual plant will act at two developmental stages: during gametophyte development (haploid gametophytic selection) and after the formation of the zygote (sporophytic selection) Hörandl (2013).

In contrast, by circumventing or suppressing meiosis and syngamy, asexual organisms skip the alternation of generation cycle and hence elude the ploidy-phase change step. In angiosperms, asexually-derived individuals can be formed either as consequence of vegetative propagation, or of asexual seed formation (apomixis), a trait that is taxonomically widespread in plants (Hojsgaard et al., 2014a). While the first involves extra vegetative growth and fragmentation without undergoing the single-cell stage and embryogenesis, the latter comprises the development of a new organism out of an unreduced, unfertilized egg cell, embryogenesis and seed formation (Mogie, 1992). In apomictic plants, a combination of complex developmental features avoid recombination and reductional steps present in the normal sexual reproductive process, thus developing a seed carrying a clonal embryo (Asker and Jerling, 1992).

A central fact for genome evolution, however, is that apomixis in angiosperms is rarely obligate. Apomictic plants produce asexual and sexual progeny within the same offspring generation, i.e., from different ovules and seeds in the same mother plant, 

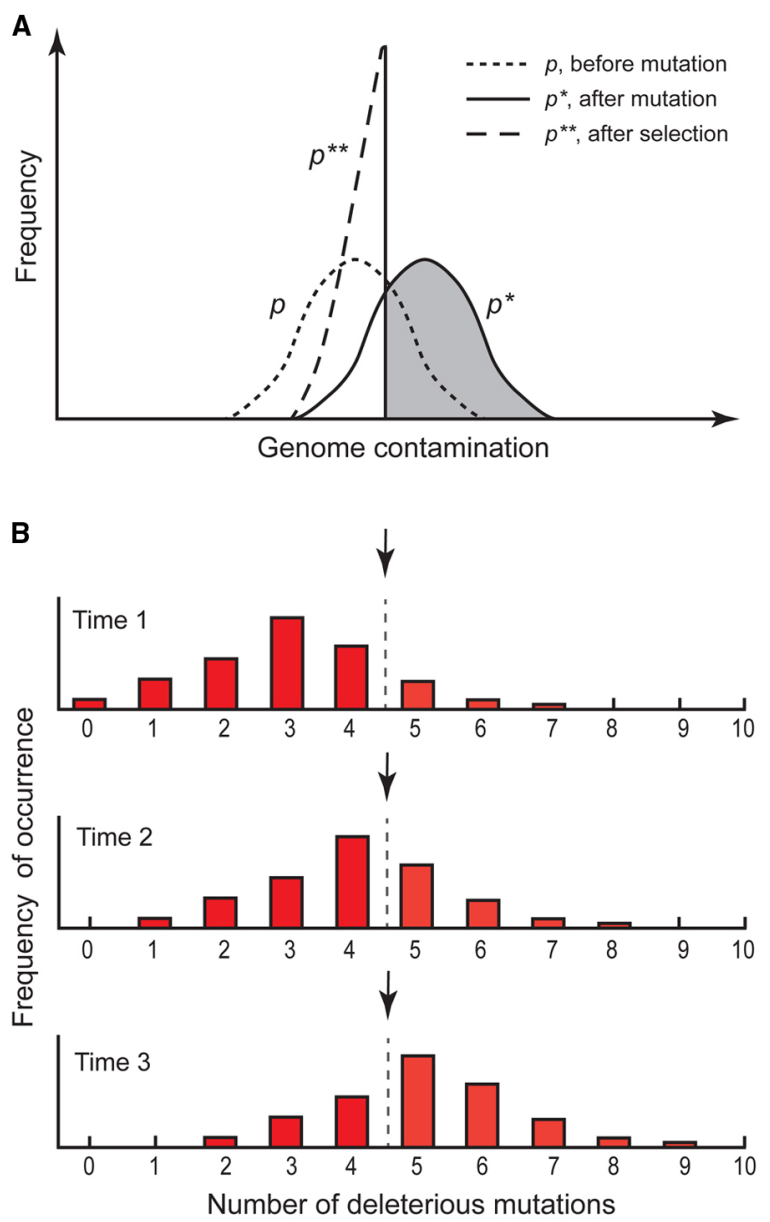

FIGURE 1 | Principles of Muller's ratchet. (A) Scheme of distributions ( $p$ ) of mutations in a sexual population. Before mutation, distribution in the population is $(p)$, after mutation, distribution shifts upward to $p^{*}$. After recombination and selection against mutants, individuals in the gray part remain sterile and die, and the distribution goes backward to $p^{* *}$. At equilibrium the means of $p$ and $p^{* *}$ are equal (redrawn after Kondrashov, 1988). (B) Scheme of mutational load distributions in an asexual population. Initially, genotypes with zero mutations exist in the population, but are lost over time by drift. Without recombination, the class with zero or few mutations cannot be restored, and consequently mutations accumulate until a threshold level of extinction (arrow) is reached (redrawn after Maynard Smith, 1988).

and therefore asexuality is facultative. Consequently, a proportion of the offspring represents recombinants, but frequencies of sexuality vary a lot among genera, species and different modes of apomixis (e.g., Aliyu et al., 2010; Sartor et al., 2011; Šarhanová et al., 2012; Noyes and Givens, 2013; Hojsgaard et al., 2013, 2014a). The role that facultative sexuality and genetically highly diverse apomictic populations play in the evolution of angiosperms is still unclear.

\section{THEORETICAL SIGNIFICANCE OF APOMIXIS FOR THE EVOLUTION OF THE PLANT GENOME}

Sexuality has the effect that deleterious mutations appear in various genotypic configurations in the offspring. Thus, harmful mutations expressed under different states (e.g., homozygous, dominant heterozygous, etc.) will negatively affect the genotype's fitness and natural selection will remove such genotypes and purge the lineage from an increase in the mutational load. Evolutionary benefits of purging a lineage from mutation accumulation have long been seen as a major advantage of sexuality (Muller, 1964; Kondrashov, 1988; Figure 1A). Apomictic plants, by circumventing genetic reshuffling mechanisms, inherit the same genomic features of their female parental genome. Without recombination, once a genotype acquire a spontaneous mutation cannot reconstitute a non-mutated genotypic state (Figure 1B and 2). Thus, a deleterious mutation in any asexual individual will be transmitted to all the offspring and loaded onto the gene pool of that clonal lineage. In any segment of a genome with absence of recombination, the number of random independent mutations is expected to increase because a mutational load smaller than the least-loaded lineage can never be generated (the ratchet mechanism, Muller, 1964; the hatchet mechanism, Kondrashov, 1988). Over time, drift will ultimately lead to a loss of genotypes with a lower mutational load and, once a threshold on mutational load is reached, to extinction of the asexual lineage (Figure 1B; Kimura et al., 1963; Muller, 1964; Felsenstein, 1974). Moreover, genetic interference effects between loci (Hill-Robertson effect, Hill and Robertson, 1966) may increase effects of mutations (Kondrashov, 1988). The genetic load of clonal lineages will reduce their fitness and obstruct further adaptation, driving those lineages to an early extinction (Maynard Smith, 1978; Bell, 1982).

Apomixis in plants is nearly exclusive associated polyploids, and often it is a result of hybridity (e.g., Koch et al., 2003; Paun et al., 2006a; Pellino et al., 2013). Polyploidy can accelerate mutation accumulation since additional gene copies represent additional mutational sites. Effects of deleterious mutations will reduce the mean fitness of any individual (and ultimately of the population) with a rate $\mathrm{c} U$, where $\mathrm{c}$ stand for the ploidy level and $U$ for the mutation rate per haploid genome (Gerstein and Otto, 2009). However, the effects of single deleterious recessive mutations in heterozygous states can be masked by a functional gene copy of the wild or dominant allele in a diploid organism (Crow and Kimura, 1970; Kondrashov and Crow, 1991; Otto and Whitton, 2000). After a prolonged diploid stage, the return to haploidy leads to the expression of accumulated, but previously masked deleterious recessive alleles, and selection against mutations (Crow and Kimura, 1970; Hörandl, 2009). However, in a polyploid plant with more than two allele copies per locus, accumulated recessive mutations in heterozygous states may still be masked after a return to haploidy. Thus, masking effects will be stronger and recessive mutations may not be effective unless they show certain level of dominance (i.e., partial dominance). So far few genomic studies are available to understand the forces underlying mutation dynamics in polyploid plants. In theory, absence of haploid gametophytic selection plus masking of recessive alleles in polyploid condition should increase the mutational load compared to sexuals.

Allelic sequence divergence, the so-called Meselson effect, is another consequence of long-term asexuality (Mark Welch and Meselson, 2000). Asexual seed reproduction, by suppress- 
ing meiosis promotes the divergence between allelic sequences by neutral mutations (Kimura and Crow, 1964). Due to the loss of sexuality, alleles within lineages will gain neutral differences at a much higher rate than the normal substitution rates observed between alleles in sexual populations (Birky, 1996). Until now, the presence of genome-wide allele sequence divergence significantly larger than those in inter-lineages have been hard to prove. Processes such as gene conversion, mitotic recombination, efficient DNA repair, meiotic parthenogenesis in animals (automixis), occasional sex, ploidy reduction, and hybridization can moderate or remove sequence divergence (e.g., Schön and Martens, 1998; Schaefer et al., 2006; Liu et al., 2007; Mark Welch et al., 2008; Flot et al., 2013). Effects of facultative sexuality on allelic sequence divergence in plants remain unknown.

\section{IS A LITTLE BIT OF SEX SUFFICIENT TO AVOID MUTATION ACCUMULATION?}

A recent transcriptome study of the Ranunculus auricomus complex, a system of diploid sexual species and hexaploid apomictic hybrids, in fact showed Meselson-like sequence divergence effects, but data did not support the idea of mutation accumulation (Pellino et al., 2013). Analyses of Muller's ratchet on high-quality single nucleotide polymorphisms (SNPs) and indels obtained from RNA-seq revealed for 1231 annotated genes that ratios of non-synonymous vs. synonymous substitutions (dN/dS) were mostly clearly below one, and did not differ significantly between apomictic-apomictic, apomictic-sexual and sexual-sexual comparisons (Pellino et al., 2013). A number of all annotated genes showed high dN/dS ratios (outlier values; see Figure 3 in Pellino et al., 2013), and hence appeared to be under divergent selection. A gene ontology analysis of outliers showed that a small proportion of those genes $(n=62 ; 6.7 \%$; Table 5 in Pellino et al., 2013) were associated with processes involved in meiosis and gametogenesis. Strikingly, most such outlier genes $(n=41$; $66.1 \%$ ) were found in the apomictic-sexual comparison and thus indicated a significant enrichment of genes associated to reproductive shifts during ovule development when compared to those outliers in sexual (or apomictic) genomes. Whether these mutations have positive or negative effects, needs further investigations. However, most mutations that are under selection do have strongly deleterious effects (e.g., Loewe and Hill, 2010). Since we analyzed only RNA sequences we assume that the observed non-synonymous substitutions mostly have negative effects (dominant or partial dominant). If deleterious mutations would have accumulated genome-wide in the apomicts-a situation expected following the nature of the genetic code and transition/transversion rates (e.g., Yang and Bielawski, 2000)they would drive the $\mathrm{dN} / \mathrm{dS}$ ratios over 1 . One interpretation of such results is that apomicts in Ranunculus are evolutionarily too young (c. 70,000 years) to have accumulated significant mutations and hence $\mathrm{dN} / \mathrm{dS}$ ratios are still similar to those in sexual putative parentals.

The alternative explanation, however, assumes that apomicts do not accumulate genome-wide deleterious mutations because facultative sexuality purges deleterious mutations (Figure 2). Detailed developmental studies revealed that apomictic hexaploid
Ranunculus hybrids show varying proportions of sexually formed seed in all genotypes, with a grand mean of 29.1\% (Hojsgaard et al., 2014b). Reduced seed set and lower pollen quality of apomicts compared to sexuals (Hörandl, 2008; Hojsgaard et al., 2014b) indicate negative effects of apomixis on fitness parameters. Population genetic studies data indicate considerable genetic diversity within and among populations (Paun et al., 2006a). Considering the expected turn-over of recombinant individuals in natural populations, regular sexuality in facultative apomicts can purge mutations via two mechanisms: First, ploidy reduction in a diploid plant can already unmask recessive deleterious mutations in the gametophyte and expose it to purging selection (Hörandl, 2009), while in a polyploid mutations showing partial dominance would be exposed to selection during haploidy (see Figure 2, stages 1 and 2); since the gametophytes represent few-celled mini-organisms, a high proportion of the genome is expressed and exposed to selection at this stage. In fact, proportions of sexual development decrease during haploid gametophyte development (Hojsgaard et al., 2013, 2014a). Second, via recombination, mutations will segregate and offspring with variable mutational load will be formed. Additionally, self-fertilization will generate zygotes with higher doses (e.g., hemizygous) of partially dominant mutated alleles, and consequently mutations will become "unmasked" and fully exposed to purging selection in the offspring (see Figure 2, stage 3). Thus, those genotypes where deleterious mutations are being expressed will be eliminated upon dosage level and only individuals carrying mutated alleles at low dosages will persist in the population (see Figure 2, stage 4 ). The lineage will consequently be regularly purged by eliminating genotypes carrying these mutations. Hence, novel mutations in a facultative will add up slower than in obligate apomicts in which each mutation is added to the mutational load (Figure 2, stages 5 and 6).

The ultimate efficacy will certainly depend on the level of functional meiosis and sexuality occurring in the population. Besides this, purging is expected to be even more efficient under diverse conditions. For example, depending on the level of penetrance and dominance of the mutation, purging would be faster as phenotypic effects would become exposed to natural selection at different dosages. A higher purging efficacy is expected with inbreeding (e.g., Agrawal and Chasnov, 2001). In fact, in a clonal population established from an apomictic mother, all neighboring individuals will carry the same deleterious mutation in their gametes. Occasional facultative sex among individuals will occur among the same genotypes, which is possible because of self-compatibility of apomictic plants (including hexaploid Ranunculus; Hörandl, 2008, 2010). Hence, plants effectively conduct self-fertilization, even if crosspollination takes place among individuals (clone-mates); thus, alleles carrying deleterious mutations may rapidly increase in their dosage, and consequently their effects will be exposed to selection (Figure 2).

Another mechanism to increase efficacy of selection can be assumed from epistasis. If additional (recessive or non-recessive) deleterious mutations lead to a larger decrease of fitness because of negative interactions of these genes, then even truncating selec- 


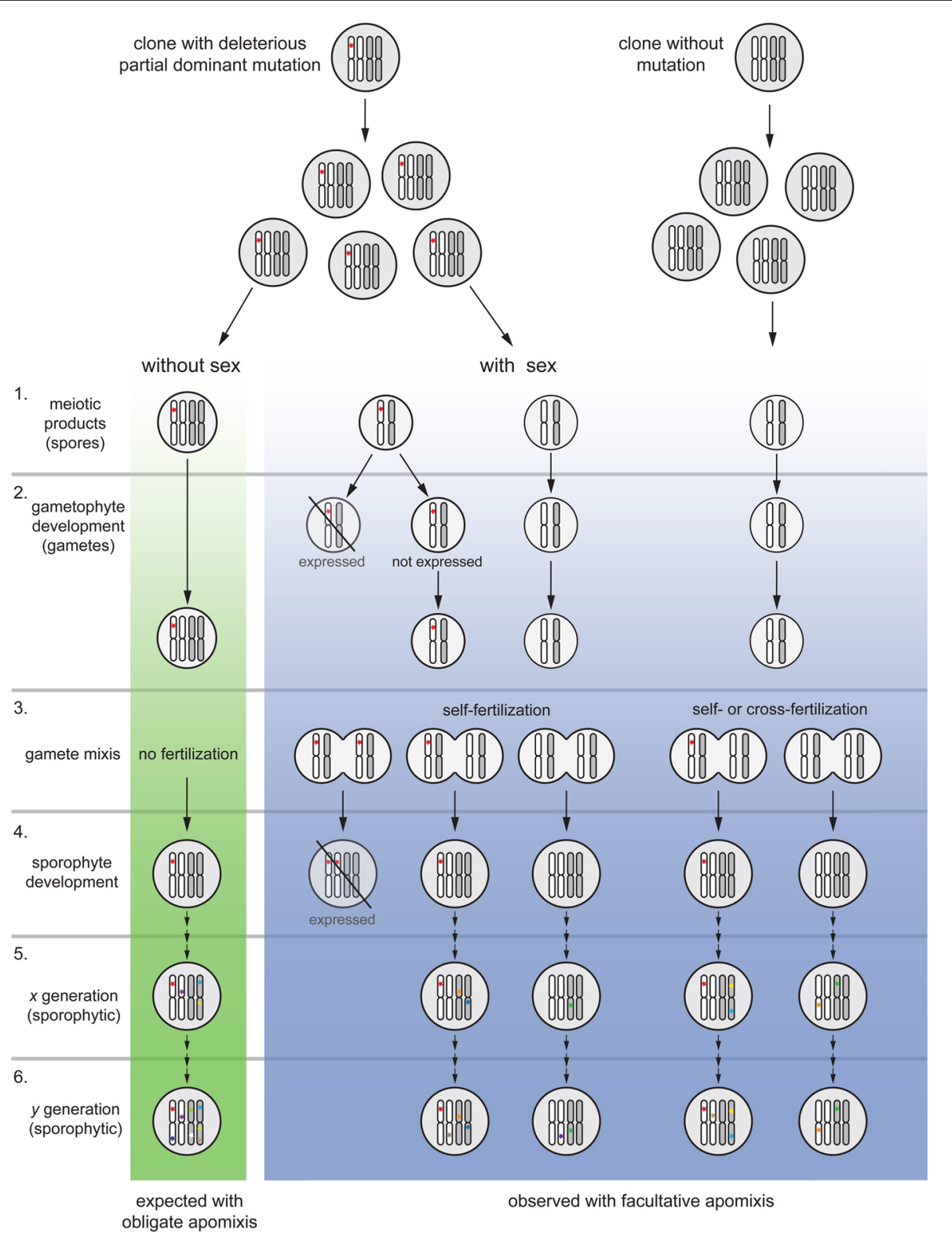

FIGURE 2 | Model of purging mutations in a tetraploid, facultative apomictic plant lineage (blue column) compared to an obligate apomict (without meiosis; green column). For simplicity, the model is presented for a new self-fertile allotetraploid lineage with regularly reduced male gametes; and partial dominant mutations are considered to be deleterious and expressed a $50 \%$ penetrance. Moreover, preferred homolog pairing is assumed during meiosis I (e.g., Comai, 2005) and considers only the perspective of a mutated deleterious allele, all other alleles pondered to be functionally equivalent. The effects of absence or presence of meiosis on mutation accumulation are illustrated after one generation following the occurrence of mutation (stages 1-4), and after several generations of obligate (without sex) or facultative apomixis (with residual levels of sex; stages 5-6). 1. Once a deleterious mutation (red star) with a $50 \%$ penetrance is loaded onto the clonal offspring, without sex only unreduced female gametes rise (clonal) progeny. With sex recombinant spores are formed. 2. Expression of mutated alleles and deleterious effects would appear only in those gametophytes with a ploidy-phase change; thus, $50 \%$ of haploid gametes would be eliminated, biasing expected progeny proportions (but not progeny types). 3. During gamete mixis, parthenogenetic embryo development avoids egg-cell fertilization in apomictic female gametophytes while meiotic ones can produce an array of progeny types upon self- or cross-fertilization syndromes. 4. A dosage increase (to duplex condition) and full expression of deleterious effects is expected in some recombinant offspring during sporophyte development, but not in non-recombinant ones. Only individuals carrying a low allele dosage (simplex condition) will remain in the population together with those without the mutation. 5-6. After a number of generations, mutations will gradually appear and added up to the genetic load in the obligate apomictic lineage. In the facultative apomictic lineage, occasional sex will segregate mutated alleles and purging selection will eliminate gametophytes and sporophytes with certain allelic dosages (as in stages 1-4). On the long run, an obligate apomictic genotype (left) will become sooner extinct compared to a facultative apomictic lineage which is continuously purged. The model does not yet consider possible purging effects via conversion during meiosis, and does not quantify facultative sexuality and actual frequencies of spore formation. The model fits to higher ploidy levels if the same penetrance level is assumed in mutated alleles. Assorted colored mutations represent independent events arisen randomly in the genome at different times. 
tion can act and rapidly eliminate this genotype (Kondrashov, 1988). Hence, despite nearly-obligate apomictic clones within a population could accumulate mutations for some generations, fitter recombinant genotypes with a lower mutational load will continuously replace them (see Figure 2). Population genetic data on Ranunculus strongly support this hypothesis of clonal turnover (Paun et al., 2006b). Consequently, Muller's ratchet is halted or at least slowed down for the lineage as whole. A fourth possibility to avoid mutation accumulation is a large effective population size (Kondrashov, 1988) and/or high migration rates amid populations (Whitton et al., 2008). Hence, within populations genetic variability will be kept at high levels and selection will act on different genotypes. Large geographical distribution and clonal diversity of the hexaploid hybrids (see Paun et al., 2006a,b) suggest that also this factor contribute to genome evolution in Ranuncu lus.

The evolutionary consequences of facultative apomixis in plants have so far received little attention. Mendelian genetic studies on control mechanism of apomixis in angiosperms suggest that the apomixis-controlling genomic regions occur -in generalin a heterozygous state (Ozias-Akins and van Dijk, 2007). In Ranunculus auricomus, quantitative expression of apomixis is dosage-dependent on the apospory factor (A), which is a dominant Mendelian factor with variable penetrance in the sporophyte, but with lethal effects in haploid or homozygous states (Nogler, 1984). Consequently, (A) appears always heterozygous with the wild type allele a in various allelic configurations, which means that apomixis cannot become completely obligate (Nogler, 1984). The long term effects of facultative sexuality remain to be studied. Overall, data (Pellino et al., 2013) suggest that apomictic polyploid lineages on the one hand accumulate Meselson-effectlike neutral substitutions in divergent gene copies, and on the other hand, mask partially dominant deleterious alleles in clones, which may become exposed to purifying selection via facultative sexuality. Comprehensive empirical studies will be needed to further test theoretical models and answer the question of how much and to what extend "a little bit of sex" protects apomictic plants from genomic decay and extinction.

\section{ACKNOWLEDGMENTS}

We thank TF Sharbel and J.-F Flot for insightful discussions. For financial support we thank the Austrian Research Foundation (FWF) under project I 310-B16 (to EH).

\section{REFERENCES}

Agrawal, A. F., and Chasnov, J. R. (2001). Recessive mutations and maintenance of sex in structured populations. Genetics 158, 913-917.

Aliyu, O. M., Schranz, M. E., and Sharbel, T. (2010). Quantitative variation for apomictic reproduction in the genus Boechera (Brassicaceae). Am. J. Bot. 97, 1719-1731. doi: 10.3732/ajb.1000188

Asker, S., and Jerling, L. (1992). Apomixis in Plants. Boca Raton: CRC press.

Bell, G. (1982). The Masterpiece of Nature: The Evolution and Genetics of Sexuality. Berkeley: University of California Press.

Birdsell, J. A., and Wills, C. (2003). The evolutionary origin and maintenance of sexual recombination: a review of contemporary models. Evol. Biol. 33, 27-138. doi: 10.1007/978-1-4757-5190-1_2

Birky, C. W. (1996). Heterozygosity, heteromorphy, and phylogenetic trees in asexual eukaryotes. Genetics 144, 427-437.

Comai, L. (2005). The advantages and disadvantages of being polyploid. Nat. Rev. Genet. 6, 836-846.
Crow, J. F., and Kimura. M. (1970). An Introduction to Population Genetics Theory. New York: Harper and Row.

Felsenstein, J. (1974). The evolutionary advantage of recombination. Genetics 78, 737-756.

Flot, J.-F., Hespeels, B., Li, X., Noel, B., Arkhipova, I., Danchin, E. G. J., et al. (2013). Genomic evidence for ameiotic evolution in the bdelloid rotifer Adineta vaga. Nature 500, 453-457. doi: 10.1038/nature12326

Gerstein, A. C., and Otto, S. P. (2009). Ploidy and the causes of genomic evolution. J. Hered 100, 571-581. doi: 10.1093/jhered/esp057

Hill, W. G., and Robertson, A. (1966). The effect of linkage on limits to artificial selection. Genetic Res. 8, 269-294. doi: 10.1017/S0016672300010156

Hojsgaard, D. H., Martinez, E. J., and Quarin, C. L. (2013). Competition between meiotic and apomictic pathways during ovule and seed development results in clonality. New Phytol. 197, 336-347. doi: 10.1111/j.1469-8137.2012.04381.x

Hojsgaard, D. H., Klatt, S., Baier, R., Carman, J. G., and Hörandl, E. (2014a). Taxonomy and biogeography of apomixis in angiosperms and associated biodiversity characteristics. Crit. Rev. Plant Sci. 33, 1-14. doi: 10.1080/07352689. 2014.898488

Hojsgaard, D. H., Greilhuber, J., Pellino, M., Paun, O., Sharbel, T. F., and Hörandl, E. (2014b). Emergence of apospory and bypass of meiosis via apomixis after sexual hybridisation and polyploidization. New Phytol. 204, 1000-1012. doi: 10.1111/nph.12954

Hörandl, E. (2008). Evolutionary implications of self-compatibility and reproductive fitness in the apomictic Ranunculus auricomus polyploid complex (Ranunculaceae). Int. J. Plant Sci. 169, 1219-1228. doi: 10.1086/591980

Hörandl, E. (2009). A combinational theory for maintenance of sex. Heredity 103, 445-457. doi: 10.1038/hdy.2009.85

Hörandl, E. (2010). The evolution of self-fertility in apomictic plants. Sex. Plant Reprod. 23, 73-86. doi: 10.1007/s00497-009-0122-3

Hörandl, E. (2013). "Meiosis and the paradox of sex in nature," in Meiosis, eds C. Bernstein and H. Bernstein (Rijeka: InTech-Open Access Publisher), 17-39. doi: $10.5772 / 56542$

Joseph, S. B., and Kirkpatrick, M. (2004). Haploid selection in animals. Trends Ecol. Evol. 19, 592-597. doi: 10.1016/j.tree.2004.08.004

Kimura, M., and Crow, J. F. (1964). The number of alleles that can be maintained in a finite population. Genetics $49,725-738$.

Kimura, M., Maruyama, T., and Crow, J. F. (1963). The mutation load in small populations. Genetics 48, 1303-1312.

Koch, M. A., Dobeš, C., and Mitchell-Olds, T. (2003). Multiple hybrid formation in natural populations: concerted evolution of the Internal Transcribed Spacer of nuclear ribosomal DNA (ITS) in North American Arabis divaricarpa (Brassicaceae). Mol. Biol. Evol. 20, 338-350. doi: 10.1093/molbev/msg046

Kondrashov, A. S. (1988). Deleterious mutations and the evolution of sexual reproduction. Nature 336, 435-440. doi: 10.1038/336435a0

Kondrashov A. S., and Crow, J. F. (1991). Haploidy or diploidy: which is better? Nature 351, 314-315. doi: 10.1038/351314a0

Liu, Q. L., Varghese, P. T., and Williamson, V. M. (2007). Meiotic parthenogenesis in a root-knot nematode results in rapid genomic homozygosity. Genetics 176, 1483-1490. doi: 10.1534/genetics.107.071134

Loewe, L., and Hill, W. G. (2010). The population genetics of mutations: good, bad, and indifferent. Phil. Trans. Roy. Soc. B 365, 1153-1167. doi: 10.1098/rstb. 2009.0317

Mark Welch, D. B., Mark Welch, J. L., and Meselson, M. (2008). Evidence for degenerate tetraploidy in bdelloid rotifers. Proc. Natl. Acad. Sci. U.S.A. 105, 5145-5149. doi: 10.1073/pnas.0800972105

Mark Welch, D., and Meselson, M. (2000). Evidence for the evolution of bdelloid rotifers without sexual reproduction or genetic exchange. Science 288, 12111215. doi: 10.1126/science.288.5469.1211

Maynard Smith, J. (1978). The Evolution of Sex. Cambridge: Cambridge University Press.

Maynard Smith, J. (1988). "The evolution of recombination” in The Evolution of Sex. An Examination of Current Ideas, eds R.E. Michod and B.R. Levin (Sinauer, Sunderland, MA), 106-125.

Mogie, M. (1992). The Evolution of Asexual Reproduction in Plants. London: Chapman and Hall.

Muller, H. J. (1964). The relation of recombination to mutational advance. Mutat. Res. 106, 2-9. doi: 10.1016/0027-5107(64)90047-8

Nogler, G. A. (1984). Genetics of apospory in apomictic Ranunculus auricomus: 5. Conclusion. Bot. Helv. 94, 411-423. 
Noyes, R. D., and Givens, A. D. (2013). Quantitative assessment of megasporogenesis for the facultative apomicts Erigeron annuus and Erigeron strigosus (Asteraceae). Int. J. Plant Sci. 174, 1239-1250. doi: 10.1086/673243

Otto, S. P. (2009). The evolutionary enigma of sex. Am. Nat. 174, S1-S14. doi: $10.1086 / 599084$

Otto, S. P., and Whitton, J. (2000). Polyploidy incidence and evolution. Annu. Rev. Genet. 34, 401-437. doi: 10.1146/annurev.genet.34.1.401

Ozias-Akins, P., and van Dijk, P. J. (2007). Mendelian genetics of apomixis in plants. Ann. Rev. Genetics 41, 509-537. doi: 10.1146/annurev.genet.40.110405.090511

Paun, O., Stuessy, T. F., and Hörandl, E. (2006a). The role of hybridization, polyploidization and glaciation in the origin and evolution of the apomictic Ranunculus cassubicus complex. New Phytol. 171, 223-236. doi: 10.1111/j.1469-8137. 2006.01738.x

Paun, O., Greilhuber, J., Temsch, E., and Hörandl, E. (2006b). Patterns, sources and ecological implications of clonal diversity in apomictic Ranunculus carpaticola (Ranunculus auricomus) complex, Ranunculaceae. Mol. Ecol. 15, 897-930. doi: 10.1111/j.1365-294X.2006.02800.x

Pellino, M., Hojsgaard, D., Schmutzer, T., Scholz, U., Hörandl, E., Vogel, H., et al. (2013). Asexual genome evolution in the apomictic Ranunculus auricomus complex: examining the effects of hybridization and mutation accumulation. Mol. Ecol. 22, 5908-5921. doi: 10.1111/mec.12533

Šarhanová, P., Vašut, R. J., Dančák, M., Bureš, P., and Trávníček, B. (2012). New insights into the variability of reproduction modes in European populations of Rubus subgen. Rubus: how sexual are polyploid brambles? Sex. Plant Reprod. 25, 319-335. doi: 10.1007/s00497-012-0200-9

Sartor, M. E., Quarin, C. L., Urbani, M. H., and Espinoza, F. (2011). Ploidy levels and reproductive behaviour in natural populations of five Paspalum species. Plant Syst. Evol. 293, 31-41. doi: 10.1007/s00606-011-0416-4
Schaefer, I., Domes, K., Heethoff, M., Schnider, K., Schön, I., Norton, R. A., et al. (2006). No evidence for the "Meselson effect" in parthenogenetic oribatid mites (Oribatida, Acari). J. Evol. Biol. 19, 184-193. doi: 10.1111/j.1420-9101.2005. 00975.x

Schön, I. and Martens, K. (1998). Opinion: DNA-repair in ancient asexuals: a new solution to an old problem? J. Nat. Hist. 32, 943-948.

Whitton, J., Sears, C. J., Baack, E. J., and Otto, S. P. (2008). The dynamic nature of apomixis in the angiosperms. Int. J. Plant Sci. 169, 169-182. doi: 10.1086/523369

Yang, Z., and Bielawski, J. P. (2000). Statistical methods for detecting molecular adaptation. Trends Ecol. Evol. 15, 496-503. doi: 10.1016/S0169-5347(00) 01994-7

Conflict of Interest Statement: The authors declare that the research was conducted in the absence of any commercial or financial relationships that could be construed as a potential conflict of interest.

Received: 31 October 2014; accepted: 01 February 2015; published online: 20 February 2015.

Citation: Hojsgaard D and Hörandl E (2015) A little bit of sex matters for genome evolution in asexual plants. Front. Plant Sci. 6:82. doi: 10.3389/fpls.2015.00082

This article was submitted to Plant Evolution and Development, a section of the journal Frontiers in Plant Science.

Copyright $(2) 2015$ Hojsgaard and Hörandl. This is an open-access article distributed under the terms of the Creative Commons Attribution License (CC BY). The use, distribution or reproduction in other forums is permitted, provided the original author(s) or licensor are credited and that the original publication in this journal is cited, in accordance with accepted academic practice. No use, distribution or reproduction is permitted which does not comply with these terms. 